\title{
O PÊSO DO CORAÇÃO EM CHAGÁSICOS CRÔNICOS *
}

\author{
J. E. H. Pitella $* *$ A. J. A. Barbosa $* *$ W. L. Tafuri $* * *$ e E. Chapadeiro $* * * *$
}

Os AA. estudaram o pêso do coração em 740 chagásicos crônicos. O pêso do coração de individuos que faleceram com e sem $I$. C. C. e sem "megas" variou de 100 a $1.075 \mathrm{~g}$. O maior número (564) era de coraçóes com I. C. C. e pesavam entre 201 e $800 \mathrm{~g}$, e apenas 45, sem I. C .C., pesavam entre 151 e $500 \mathrm{~g}$.

o pèso do coração de individuos que faleceram com e sem I. C. C. mas com "megas" variou de 100 a $750 \mathrm{~g}$. O maior número de coraçóes com (39) $e$ sem (60) I. C. C. pesou entre 101 e $450 \mathrm{~g}$.

o pêso médio dos corações chagásicos (740) foi de $435 \pm 5,6 \mathrm{~g}$. Já o pêso médio dos corações chagásicos com e sem I. C. C., sem "megas' foi de $479 \pm 5,6$ $g$ e o pêso médio dos coraçóes com e sem I. C. C. e com "megas" foi de $314 \pm 12,6$ g. A análise estatistica aplicada demonstrou haver diferença significativa entre as duas médias.

A hipotrofia constatada nos coraçôes we individuos portadores de "megas", especialmente de megaesôfago, está relacionada com o estado de desnutrição $e$ não com a idade.

O pêso e o volume do coração no chagásico crônico variam enormemente. Segundo observações de Mignone (5), Raso (6) Andrade e Andrade (1), Lopes e col. (1) e outros, na doença de Chagas podem-se encontrar grandes e pequenas cardiomegalias ao lado de coraçóes com pêso normal, ou até mesmo hipotróficos. Lopes e col.(1) demonstraram também que o pêso do coração de chagásico crônico varia de acôrdo com o tipo de morte apresentado pelo paciente.

Com a finalidade de ampliar os dados estatísticos já existentes e comparar os nossos resultados com os do trabalho de Lopes e col. (4), fizemos um levantamento dos casos de cardiopatia chagásica dentre 15.000 necrópsias realizadas no Departamento de Anatomia Patológica da Faculdade de Medicina da Universidade Federal de Minas Gerais.

\section{MATERIAL E MÉTODOS}

Consta de 946 corações, sendo 206 de individuos sadios (grupo I) retirados dentre 230 nas condições explicadas em trabalho anterior de Tafuri e Chapadeiro(i), os

* Trabalho do Departamento de Anatomia Patológica (Prof. L. Bogliolo), da Faculdade de Medicina da UFMG.

* Bolsistas da CAPES.

** Frof. Adjunto e Chefe de Pesquisa do CNPq. **** Prof. Titular da Cadeira de Patologia da Faculdade de Medicina do Triângulo Mineiro (Uberaba).
Recebido pana publicaçåo em 30_3-70. 
quais serviram de contrôle. Os 740 corações restantes, de adultos, que tinham o seu pêso registrado no protocolo de necrópsia, foram retirados dentre 875 casos de chagásicos crônicos nas 15.000 necrópsias realizadas. Dentre êstes, 115 apresentavam a associação "megas"-cardite crônica. Por esta razão e pensando na possibilidade de modificação do pêso do coração devido ao "mega", foram feitas duas tabelas (Tabelas I e II) nas quais foi analisado o pêso do coração de pacientes chagásicos sem "mega", com e sem insuficiência cardíaca congestiva (I. C .C., Tab. I) e o pêso do coração com "mega", com e sem I. C. C. (Tab. II).

Os 740 coraçoes foram ainda divididos em 7 grupos, a saber: grupo II (número total dos corações chagásicos com e sem "megas", com e sem I .C. C.); grupo III (número de corações chagásicos com e sem I. C. C. e sem "megas"); grupo IV (número de coraçôes chagásicos com I. C. C e sem "megas"); grupo V (número total de corações chagásicos sem I. C .C. e sem "megas"); grupo VI (número de corações chagásicos com e sem I. C. C. e com "megas") ; grupo VII (número de coraçôes chagásicos com I. C. C. e com 'megas') e grupo VIII (número de corações chagásicos sem I. C. C. e com "megas"). Todos êstes grupos estão contidos e analisados na tabela III.

Todos os corações foram retirados de acôrdo com a técnica de Franco (").

\section{RESULTADOS}

$\mathrm{Na}$ tabela I estão distribuídos os coracões chagásicos de indivíduos com e sem I. C. C. e sem "megas" em relação ao pêso, que variou de 100 a $1.075 \mathrm{~g}$. Do total (625), 579 corações eram de pacientes chagásicos que faleceram com I. C. C. e 46 sem o quadro anatomopatológico de I. C. C. Verificase também que o maior número (564) de corações com I. C. C. pesou ențre 201 a $800 \mathrm{~g}$ e o de corações sem I. C .C. (45) pesou entre 151 a $500 \mathrm{~g}$. Acima de $800 \mathrm{~g}$ encontram-se 13 corações chagásicos com I. C. C. e nenhum sem I. C. C. Nos que pesaram menos de $151 \mathrm{~g}$ encontrou-se 1 (um) sem I. C. C. e nenhum com I. C. C.

$\mathrm{Na}$ tabela II estão distribuídos os corações chagásicos de inđividuos com e sem
I. C. C. e com "megas" em relação ao pêso que variou de 100 a 750 g. Do total 1151, 55 corações eram de pacientes chagásicos com I. C. C. e 60 sem I. C. C. Vê-se que o maior número de corações com (39) e sem (60) I. C. C. pesou entre 101 a $450 \mathrm{~g}$.

$\mathrm{Na}$ tabela III estão distribuídas as médias dos pêsos dos diferentes grupos, conforme explicado antes. A comparação entre as médias dos pesos dos coraçōes contrôles (grupo I) e dos chagásicos (grupo II) mostra que a do último é maior do que a do contrôle. O teste de $t$ demonstrou existir uma diferenca altamente significativa $(t=13,0)$. O mesmo se verifica em relação ao grupo IV (coraçōes de chagásicos que morreram com I. C. C.) $t=17,43$. Pelo contrário, as médias dos grupos V, VI e VII estão próximas ou mesmo inferiores às do grupo contrôle. O teste de $t$ aplicado entre as médias do grupo contrôle (grupo I) e as dos grupos V, VI e VII não demonstrou existir diferença estatisticamente significativa. Todavia, para o grupo VIII o teste de $t(t=5,6)$ foi significativo.

\section{COMENTÁRIOS}

Analisando os nossos resultados, podese concluir o seguinte: 1) - $91,03 \%$ dos corações chagásicos com I. C. C. e sem "megas" pesavam mais de $300 \mathrm{~g}$, enquanto que $71,70 \%$ dos coraçōes chagásicos sem I. C. C. e sem "megas" pesavam mais de $300 \mathrm{~g} ; 2)-65,43 \%$ dos corações chagásicos com I. C. C. e com "megas" pesavam mais de $300 \mathrm{~g}$ enquanto que $20,00 \%$ dos corações chagásicos sem I. C. C. e com "megas" pesavam mais de $300 \mathrm{~g}$; 3) - dos coraçóes. contrôles, apenas $49,00 \%$ pesavam mais de $300 \mathrm{~g} ; 4$ ) - por outro lado, estabelecendo-se a mesma comparação com os pesos de corações acima de $500 \mathrm{~g}$, verifica-se que $35,17 \%$ do total das cardiopatias chagásicas com I. C. C. e sem "megas" pesavam mais de $500 \mathrm{~g}$, enquanto que nenhum dos coraçōes sem I C. C. pesava mais de $500 \mathrm{~g}$; 5) - a mesma diferença não ocorreu em relação à cardiopatia chagásica associada a "megas", pois apenas $19,99 \%$ dos corações com I. C. C. pesava acima de $500 \mathrm{~g}$, enquanto que nenhum dos corações sem I. C. C. pesava acima de $500 \mathrm{~g} ; 6$ ) — a tabela III 
tante diferentes quando comparados com os corações de cardiopatias sem "megas", com ou sem I. C .C. De fato, como demonstra a fig. 1, o pêso do coração chagásico, quando associado a "megas", é inferior ao do coração chagásico não associado a "megas". Há corações que são até hipotróficos, pesando menos de $200 \mathrm{~g}$. Êste resultado está de acôrdo com o de Andrade e Andrade (1) que encontraram coraçóes de até $120 \mathrm{~g}$.

A nosso ver, o coração chagásico associado a "megas" tem pêso menor do que o coração chagásico sem "mega", com ou sem I. C. C., pelas seguintes razóes: 1) a cardite é sempre de pequena intensidade, não levando, portanto, à fibrose acentuada; 2) - o portador de "mega", especialmente de megaesôfago, pela dificuldade em deglutir os alimentos depaupera-se paulatinamente e a desnutrição, às vêzes grave, que se observa nestes indivíduos seria a maior responsável pelos fenômenos regressivos (hipotrofia e degeneração) das fibrocélulas cardíacas e consecutiva diminuição do pêso e do volume do órgão. De fato, 31 ou $68.8 \%$ dos corações, dentre os 45 que tinham pêso entre 100 a 250 gramas, pertenciam a adultos jovens $(15$ a 35 anos) e adultos ( 36 a 45 anos), portadores de "megas". Para verificar se a intensidade da hipertrofia constatada no coração de indivíduos portadores de "megas" é conseqüência do estado caquético decorrente do "mega" ou se deriva de outros fatores, tomamos amostras, ao acaso, de 90 coracões que pesavam entre 100 a 250 $\mathrm{g}$, no mesmo grupo etário ( 15 a 45 anos), de pacientes que faleceram de outras causas caquetizantes e não caquetizantes. $\mathrm{Ob}-$ servamos que o maior numero $(57$ ou $63.3 \%$ ) de corações eram de indivíduos portadores de doenças caquetizantes. Êstes resultados vêm demonstrar que a hipotrofia do coração não depende da idade, mas está relacionada com o estado de desnutrição do indivíduo; 3) - menor incidência de I. C. C. $(47.7 \%)$ em relação ao

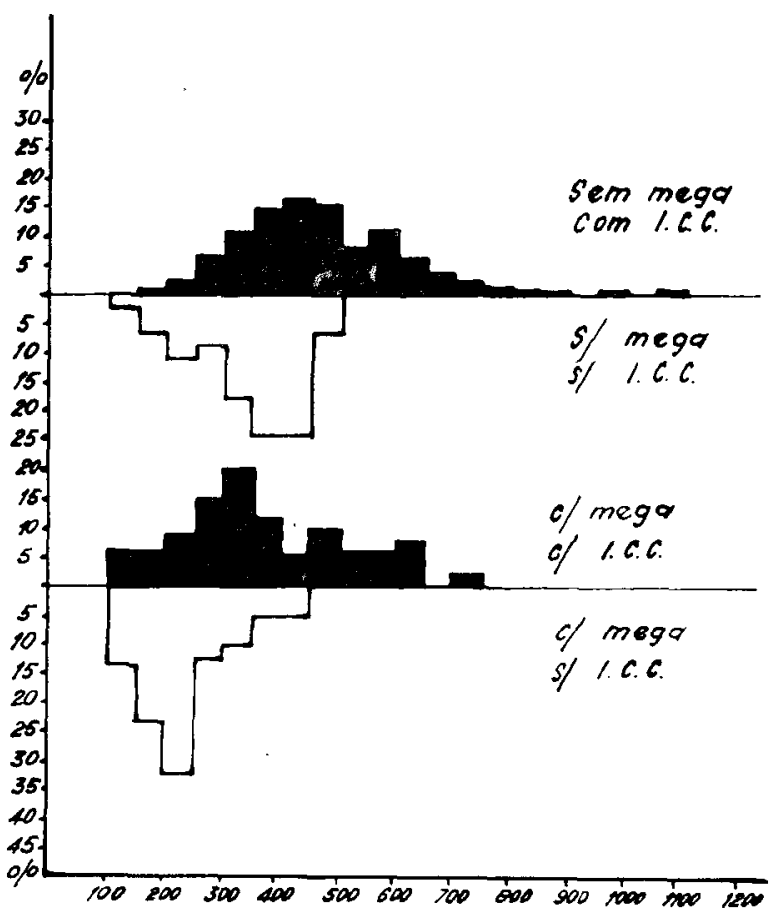

Fig. 1 - Histograma mostranco a distribuiçâo de frequiência dos pesos dos corações chagásicos com e sem "megas" com e sem I.C.C. 
TABELA II

DISTRIBUIÇÃO DE FREQU̇ÊNCIA DOS PESOS DOS CORAÇÕES CHAGÁSICOS DE INDIVIDUOS COM E SEM I. C. C. E COM "MEGAS"

\begin{tabular}{|c|c|c|c|c|}
\hline $\begin{array}{c}\text { Pêso } \\
g\end{array}$ & $\begin{array}{c}\text { N. Cardiopatias } \\
\text { C/ "Megas" } e \\
\text { C/I.C.C. }\end{array}$ & $\%$ & $\begin{array}{c}\text { N. Cardiopatias } \\
\text { C/"Megas"e } \\
\text { S/I.C.C. }\end{array}$ & $\%$ \\
\hline $100-150$ & 3 & 5,45 & 8 & 13,33 \\
\hline $151-200$ & 3 & 5,45 & 14 & 23,33 \\
\hline $201-250$ & 5 & 9,09 & 19 & 31,66 \\
\hline $251-300$ & 8 & 14,54 & 7 & 11,66 \\
\hline $301-350$ & 11 & 20,00 & 6 & 10,00 \\
\hline $351-400$ & 6 & 10,90 & 3 & 5,00 \\
\hline $401-450$ & 3 & 5,45 & 3 & 5,00 \\
\hline $451-500$ & 5 & 9,09 & & \\
\hline $501-550$ & 3 & 5,45 & & \\
\hline $551-600$ & 3 & 5,45 & & \\
\hline $601-650$ & 4 & 7,27 & & \\
\hline $651-700$ & - & 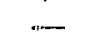 & & \\
\hline $701-750$ & 1 & 1,82 & & \\
\hline TOTAL & 55 & $\mathrm{x}$ & 60 & $\mathrm{x}$ \\
\hline
\end{tabular}

total de casos de cardiopatia chagásica sem "megas" $(92.64 \%)$. Todavia, a I. C. C., por si só, independentemente da cardite, pode concorrer, em parte, para o au- mento de pêso do coração, pois o pêso médio do coração associado a "megas" com I. C .C. foil superior ao do coração associado a "megas" sem I. C. C. (fig. 1).

TABELA III

PÊSO MÉDIO DOS CORAÇÕES CONTROLLES E DOS CHAGÁSICOS

\begin{tabular}{c|c|c|c|c|c}
\hline Grupos & N. & $\begin{array}{c}\text { Amplitude } \\
\text { Total }\end{array}$ & $\begin{array}{c}\text { Média } \pm \\
E . P .\end{array}$ & C.V. & D. Padrão \\
\hline I & 206 & $150-500$ & $310 \pm 4,2$ & 66 & 21,3 \\
II & 740 & $100-1075$ & $453 \pm 5,6$ & 33,7 & 152,9 \\
III & 625 & $145-1075$ & $479 \pm 5,6$ & 29,6 & 141,7 \\
IV & 579 & $200-1075$ & $489 \pm 5,8$ & 28,8 & 140,8 \\
V & 46 & $145-480$ & $357 \pm 12,7$ & 24,1 & 86,2 \\
VI & 115 & $100-650$ & $314 \pm 12,6$ & 43,1 & 135,4 \\
VII & 55 & $120-650$ & $383 \pm 20,1$ & 38,8 & 148,9 \\
VIII & 60 & $100-45 / J$ & $251 \pm 10,5$ & 32,3 & 81,2 \\
\hline
\end{tabular}

GRUPO I - CONTRÔLE

GRUPO II - TOTAL DOS CORAÇÕES CHAGÁSICOS C/S I.C.C. C/S MEGAS

GRUPO III - C/S I.C.C. S/MEGAS

GRUPO IV - C/I.C.C. S/MEGAS

GRUPO $\quad \mathrm{V}-\mathrm{S} /$ I.C.C. S/MEGAS

GRUPO VI - C/S I.C.C. C/MEGAS

GRUPO VII - C/I.C.C. C/MEGAS

GRUPO VIII - S/I.C.C. C/MEGAS 


\section{SUMMARY}

The authors have studied the weight of the heart in 740 chronic chagasic. The weight of the heart of people who died with or without congestive heart failure has varied from $100 \mathrm{~g}$ to $1.075 \mathrm{~g}$. The largest number (564) of those hearts were hearts with congestive heart failure and they weighed between 201 $g$ and $800 \mathrm{~g}$, but only 45 hearts which had no congestive heart failure weighed between $151 \mathrm{~g}$ and $500 \mathrm{~g}$.

The weight of the heart of people who died with or without congestive heart failure but with "megas" has varied from $100 \mathrm{~g}$ to $750 \mathrm{~g}$. The largest number of hearts with congestive heart failure (39) and without congestive heart failure (60) weighed between $101 \mathrm{~g}$ and $450 \mathrm{~g}$.

The medium weight of chagasic hearts $(740)$ was $453 \pm 5.5 \mathrm{~g}$. On the other hand, the medium weight of chagasic hearts with or without congestive heart failure and without "megas" was $479 \pm 5.6 \mathrm{~g}$ and the medium weight of hearts with or without congestive heart failure but with "megas" was $314+12.6 \mathrm{~g}$. The statistical analysis used on this purpose has shown a significant difference between the two averages.

The atrophy observed in the hearts of people with "megas" and especially of megaesofagus is connected with their malnutrition state and not with their age.

\section{BIBLIOGRAFIA}

1) ANDRADE. Z. A. e ANDRADE, S. G. - O coração nos "megas" do aparelho digestivo. O Hospital. 71: 719-726, 1067.

2) DOEHNERT, H. R. e MOTTA, G. Enfermedad de Chagas y miocarditis cronica. Arch. Venez. de Med. Trop. y Parasit. Medica. Vol. V (1): 123150, 1965.

3) FRANCO, E. E. - Manual atlas de las autopsias. Salvat Edit., S. A. Barcelona, 1. ${ }^{2}$ ed., 1929

4) IOPES, E. R.; CHAPADEIRO, E.; TAFURI, W. L.; ALMEIDA, H, O. e AERÃO, D. - Pêso do coração e tipo de morte no chagásico crônico. (Em publicação), 1970 .
5) MIGNONE, C. - Alguns aspectos da anatomia patológica da cardite chagásica crônica. Tese Prof. Catedr. de Anat. Patol. da Fac. Med. da Univ. S. Paulo. 238 pp., 1958.

6) RASO P. - Contribuicão ao estudo da lesão vorticilar especialmente do vórtex esquerdo) na cardite chagásica crónica. Tese Livre-Docência de Anat. Patol. da Fac. Med. da Univ. Fed. M. G.; Imprensa da Universidade de Minas Gerais, Belo Horizonte, 124 pp., 1964.

7) TAFURI, W. L. e CHAPADEIRO, E. - O pêso do coração no brasileiro adulto normal, O Hospital. 70: 141151, 1966 . 